\title{
Concurrent Simulation of Neuromuscular Control and Tissue Deformation is Possible by Adaptive Surrogate Modeling
}

Jason P. Halloran

Cleveland Clinic

Ahmet Erdemir

Cleveland Clinic

Antonie J. van den Bogert

Cleveland State University, a.vandenbogert@csuohio.edu

Follow this and additional works at: https://engagedscholarship.csuohio.edu/enme_facpub

Part of the Biomechanical Engineering Commons

How does access to this work benefit you? Let us know!

\section{Original Citation}

Halloran, J.P., Erdemir, A., and van den Bogert, A.J., 2009, "Concurrent Simulation of Neuromuscular Control and Tissue Deformation is Possible by Adaptive Surrogate Modeling," Proceedings of the ASME Summer Bioengineering Conference, 2008, Parts A and B, pp. 763-764.

This Conference Proceeding is brought to you for free and open access by the Mechanical Engineering Department at EngagedScholarship@CSU. It has been accepted for inclusion in Mechanical Engineering Faculty Publications by an authorized administrator of EngagedScholarship@CSU. For more information, please contact library.es@csuohio.edu. 


\title{
Concurrent musculoskeletal dynamics and finite element analysis predicts altered gait patterns to reduce foot tissue loading
}

\author{
Jason P. Halloran a, Marko Ackermann ${ }^{a}$, Ahmet Erdemir ${ }^{\text {a,b }}$, Antonie J. van den Bogert ${ }^{\text {a,* }}$ \\ a Department of Biomedical Engineering, Cleveland Clinic, 9500 Euclid Ave., Cleveland, OH 44195, USA \\ ${ }^{b}$ Computational Biomodeling Core, Cleveland Clinic, 9500 Euclid Ave., Cleveland, OH 44195, USA
}

\section{Introduction}

In musculoskeletal biomechanics, computational modeling often represents a cost-effective approach to iterate design variables of interest as well as estimate otherwise unmeasurable metrics, e.g. tissue loading. Current computational methods for simulating human movement have primarily consisted of muscledriven movement simulations (Neptune, 2000; Zajac et al., 2003; Anderson and Pandy, 2001; Erdemir et al., 2007; van den Bogert, 1994). Due to computational expense, such simulations generally include assumptions which simplify joint or soft tissue behavior. Joints are typically modeled as hinge or spherical and soft tissue effects are included as spring-dashpot systems. These limiting assumptions are serious obstacles to applications such as ligament injury or osteoarthritis, where local tissue loading must be predicted (McLean et al., 2003; Lin et al., 2009). Conversely, continuum soft tissue deformation models, generally performed

\footnotetext{
* Correspondence to: Orchard Kinetics LLC, 2217 S. Overlook Rd., Cleveland Heights, OH 44106, USA. Tel.: 2165393638.

E-mail address: bogert@orchardkinetics.com (A.J. van den Bogert).
}

using the finite element (FE) method, can provide information on local tissue loading but require assumed or measured boundary conditions (Huiskes and Hollister, 1993; Zhang et al., 1998; Jones and Wilcox, 2008). Coupling detailed deformable soft tissue and musculoskeletal models would help overcome the limitations of each modeling domain by combining their strengths.

Previously, multidomain studies have been performed but until recently these were generally non-concurrent, in which tissue deformations were analyzed by post-processing of a given musculoskeletal loading state (Besier et al., 2005; Cattaneo et al., 2005; El-Rich and Shirazi-Adl, 2005; Fernandez and Pandy, 2006; Hopkins et al., 2005). These studies lacked the coupled nature of soft tissue deformation and muscular loading. Of notable exception, Koolstra and van Eijden (2005) used an explicit FE approach to estimate mandible stresses during a forward dynamic simulation. However, computational expense of such an approach limits the framework for use in iterative analyses, i.e. optimization or probabilistic studies. More recently, we demonstrated direct coupling between a musculoskeletal lower extremity model and FE foot model to solve a maximum height jumping objective (Halloran et al., 2009). To reduce the computational cost of FE foot simulations, an adaptive surrogate modeling method was 


\begin{tabular}{|llll|}
\hline Nomenclature & $v_{x}$ & ankle horizontal velocity \\
& & $\omega_{z}$ & foot rotational velocity \\
FE & finite element & $T$ & gait cycle period \\
MTP & metatarsophalangeal & $x(t)$ & musculoskeletal time histories of model states \\
NLP & nonlinear programming & $u(t)$ & time histories of muscle neural excitations \\
RMS & root mean square & $J_{t r}$ & kinematics tracking term \\
$q$ & skeleton pose & $J_{f t g}$ & muscle fatigue term \\
$q_{F E A}$ & finite element ankle position & $J_{s t r}$ & maximal strain energy density in the plantar tissue \\
$M$ & mass matrix & $\omega_{n}$ & cost function weight values \\
$C$ & centrifugal and coriolis terms & $n_{t r}$ & number of tracked variables in tracking term \\
$G$ & gravity terms & $y_{j}$ & simulated kinematics variable \\
$F_{M T}$ & muscle forces & $\bar{y}_{j}$ & mean measured kinematics \\
$R$ & muscle moment arms & $\sigma_{j}$ & standard deviation of kinematics measurements. \\
$Q_{f r}$ & friction term & $m$ & number of muscle groups \\
$Q_{F E A}$ & finite element reaction loads & $\Phi$ & muscle fatigue function \\
$f_{z}$ & net vertical (normal) ground reaction force & $a$ & muscle activations \\
$v_{c x}$ & horizontal sliding velocity of the contact points & $\phi$ & function describing stress-strain distribution within \\
$v_{c}$ & friction term scaling factor & & plantar tissue \\
$\mu$ & friction coefficient & $n_{F E}$ & number of finite element nodes \\
$z$ & ankle vertical position & & \\
\hline
\end{tabular}

adopted, based on locally weighted regression to estimate ankle reaction loads. The study showed that it would be possible to simultaneously predict movement and tissue deformations in a coupled iterative analysis. Nonetheless, the study has limited clinical relevance, and the potential of tissue level mechanical variables to influence predicted movement was not employed.

The capability to predict adaptations in movement due to mechanical changes in tissues, or as a function of desired unloading of the tissue, has important implications for design of surgical, therapeutic and rehabilitative interventions. For example, this would provide a scientific basis for design of neuromuscular strategies to prevent anterior cruciate ligament injury (Shimokochi et al., 2009; Boden et al., 2000; Markolf et al., 2004; McLean et al., 2004). Rehabilitation programs for tissue relief could be developed for the prevention of lower back pain, osteoarthritis, or plantar tissue pressure, thought to be a contributor to diabetic foot ulceration (Veves et al., 1992). With continuum models of tissue behavior, internal conditions, such as strain, shear, and stress, could not only be quantified but utilized in the formulation of the movement objective.

The goal of the current study was twofold: first, develop a predictive neuromuscular controlled simulation of gait by coupling a finite element (FE) model of the foot with a lower limb musculoskeletal model; and second, incorporate the minimization of a peak plantar tissue deformation metric into the objective of a predictive movement optimization. The successful demonstration of this capability establishes a framework to develop future clinical applications.

\section{Methods}

\subsection{Musculoskeletal model}

Details of the musculoskeletal model have been described previously (Gerritsen et al., 1998). Briefly, the model was two-dimensional and contained seven body segments: trunk, thighs, shanks, and feet. Joints were ideal hinges, and there were no kinematic constraints between the feet and ground, resulting in a total of nine kinematic degrees of freedom. Eight muscle groups were included in each lower extremity: iliopsoas, glutei, hamstrings, rectus femoris, vasti, gastrocnemius, soleus, and tibialis anterior. Each muscle was represented by a 3-element Hill model, as described in McLean et al. (2003), with muscle properties from Gerritsen et al. (1998), and simulated with custom C code. The model had 50 state variables in $x$ : 9 generalized coordinates in $q$, 9 generalized velocities in $\dot{q}$, 16 muscle contractile element lengths in $l_{c e}$, and 16 muscle activations in $a$. Equations of motion were generated by SD/Fast (Parametric Technology Corp., Needham, MA):

$M(q) \ddot{q}+C(q, \dot{q})+R F_{M T}+G(q)+Q_{f r}(q, \dot{q})+Q_{F E A}\left(q_{F E A}(q)\right)=0$,

where $M$ is the mass matrix, $C$ are centrifugal and Coriolis effects, $G$ contains gravity terms, $F_{M T}$ are the muscle forces, applied via a matrix of moment arms $R$, and $Q_{f r}$ is the friction term (described below). The final term represents reaction loads applied to the calcaneus by the finite element model of the foot, which is introduced below. These loads are only dependent on kinematic boundary conditions $q_{\text {FEA }}$ which are a known function of skeleton pose $q$.

\subsection{Finite element model of the foot}

A plane strain foot model was implemented in Abaqus (Simulia, Providence, RI) (Halloran et al., 2009). A sagittal plane cross-section along the second ray of the foot was used to represent the bone and tissue geometry. Bones were modeled as rigid and the soft tissue as an Ogden material model with material properties based on heel pad indentation tests (Erdemir et al., 2006). Bones other than the phalanges were combined into one rigid segment, which was controlled by prescribing the vertical position and the orientation of the talus relative to the ground. These were the kinematic boundary conditions for the finite element analyses. As a result, the ankle is modeled as a hinge joint with the FE model of the foot and the musculoskeletal model sharing rigid body boundary conditions at the calcaneus. Ankle joint coordinates, $q_{F E A}$, were directly coupled between the FE and musculoskeletal models. The phalanges were represented as another rigid segment, which was free to move during simulations. Soft tissue surrounding the metatarsophalangeal (MTP) joint served to restrain the movements of this segment during passive toe flexion. Elements between the metatarsal head and the proximal phalanx also contributed to passive MTP joint stiffness and were modeled as linearly elastic $(E=1 \mathrm{e} 6 \mathrm{~Pa}, v=0.3)$. Contact between foot and ground was modeled as frictionless in the finite element analysis, but an approximate friction model was included in the musculoskeletal simulations to represent the overall shear loading on the foot, as described below. Each time the vertical position of the talus and its orientation was passed to the finite element model, the FE model was solved and vertical reaction force and moment at the calcaneus were returned to the musculoskeletal model. As friction was not included in the FE contact definition, the need to prescribe the horizontal position of the talus was unnecessary. Stress-strain distribution within the soft tissue and plantar pressures were available as additional outputs from the finite element analysis.

\subsection{Friction model}

Friction between feet and ground was not included in the FE model, but is required for normal gait. The horizontal contact force $Q_{f r}$ was modeled by an approximation of the Coulomb friction defined as a function of the net vertical (normal) ground reaction force $f_{y}$ generated by the $\mathrm{FE}$ foot model and an estimation of the horizontal, sliding velocity of the contact points $v_{c x}$ as

$Q_{f r}=-\frac{1-\exp \left(-v_{c x} / v_{c}\right)}{1+\exp \left(-v_{c x} / v_{c}\right)} \overleftarrow{\mu} f_{z}$ 
where $v_{c}$ is a scaling factor set to $v_{c}=0.05 \mathrm{~m} \mathrm{~s}^{-1}$ and $\mu$ is the friction coefficient set to $\mu=1.0$ (Ackermann and van den Bogert, 2010). Sliding velocity of the contact points $v_{c x}$ was defined as a function of ankle vertical position $z$, ankle horizontal velocity $v_{x}$, and rotational velocity of the foot $\omega_{z}$ as

$v_{c x}=v_{x}+\omega_{z} z$

\subsection{Movement prediction}

The predictive gait simulation was formulated as an optimal control problem that searches for time histories of states $x(t)$ and neural excitations $u(t)$ that are periodic, have a specified walking speed (here set at $1.1 \mathrm{~m} / \mathrm{s}$ ), satisfy the system dynamics and minimize a cost function (Ackermann and van den Bogert 2010). The following cost function was used:

$J=\omega_{1} J_{t r}+\omega_{2} J_{f t g}+\omega_{3} J_{s t r}$,

where $J_{t r}$ is a "tracking" term containing the deviation from normative gait kinematics and ground reaction data in Winter (1991), $J_{f t g}$ is a term quantifying muscle "fatigue", and $J_{s t r}$ is a term expressing maximal strain energy density in the foot tissue. Weights $\omega_{n}$ were included for each term. The "tracking" was computed as

$J_{t r}=\frac{1}{T} \frac{1}{n_{t r}} \sum_{j=4}^{n_{t r}}\left(\int_{0}^{T}\left(\frac{y_{j}(t)-\bar{y}_{j}(t)}{\sigma_{j}(t) \leftarrow}\right)^{2} d t\right.$,

where $n_{t r}$ is the number of tracked variables, $y_{j}$ is the simulated variable, $\bar{y}_{j}$ is the mean measured value of the variable, and $\sigma_{j}$ is the standard deviation of the measurements. Knee, hip, ankle angles, and vertical and horizontal ground reaction forces were tracked using published inter-subject mean and standard deviation data, and this term was included to approximate the simulated gait to normal patterns (Winter, 1991). The "fatigue" term was defined as

$J_{f t g}=\leftarrow \sum_{i=\mathcal{A}}^{m}\left(\Phi_{i}^{10}\right)^{1 / 10} \approx \operatorname{minmax}_{i} \Phi_{i}$,

where $m$ is the number of muscle groups and $\Phi$ is a measure of muscle fatigue related to the cube of muscle activation $a$ (Crowninshield and Brand, 1981)

$\Phi_{i}=\iint_{\oint}^{T} a_{i}^{3}(t) d t$.

The exponent in Eq. (6) provides a continuous approximation to the minmax problem of minimizing the maximal muscle fatigue, i.e. maximizing endurance (Rasmussen et al., 2001). This term is necessary because muscle force sharing is not uniquely determined by the tracking term in the cost function. The third term in Eq. (4) quantifies the tissue mechanical state over the gait cycle through a continuous function of the maximal strain energy density as

$J_{s t r}=\frac{1}{T} \int\left(\sum_{k=4}^{T}\left(\rho_{k}^{6}(t) d t\right)^{1 / 6}\right.$,

where $n_{F E}$ is the number of FE nodes including both feet and $\varphi$ is the strain energy density at each node in the FE foot model tissue in $\mathrm{N} / \mathrm{mm}^{2}$. The exponent of six in Eq. (8) was selected because it strongly penalizes large strain energy densities while avoiding the strong nonlinearities associated with even higher exponents.

Direct collocation was used to transform the optimal control problem into a nonlinear programming problem (NLP) which was solved in Matlab (The Mathworks, Inc., Natick, MA) using the optimization package SNOPT (Tomlab Optimization, Pullman, WA), interacting with Abaqus (Simulia, Providence, RI) for finite element analysis and custom $C$ code for musculoskeletal dynamics. Details are provided in Ackermann and van den Bogert (2010).

As per the objectives of the study, two simulations were performed. The first incorporated just the tracking and fatigue terms $\left(\omega_{3}=0\right)$ with $\omega_{1}=1$ and $\omega_{2}=10$, both set to bring the terms to the same order of magnitude. In the results, this solution is referred to as "tracking". In the second simulation, the $\omega_{1}$ and $\omega_{2}$ weights were retained and $\omega_{3}$ was set to 500, resulting in approximately equal contributions of all terms. This solution is referred to as the "strain" simulation.

\section{Results}

The optimization successfully converged for both objective functions (Fig. 1). Dynamic equilibrium and periodicity were satisfied throughout the simulations. The "tracking" solution matched desired kinematics with root mean square (RMS) values of $1.5^{\circ}, 1.5^{\circ}$, and $1.9^{\circ}$ for hip, knee, and ankle angles, respectively (Fig. 2). The physical location of peak strain energy density occurred at progressive locations in the plantar tissue throughout the cycle from the heel to the toe throughout the cycle. Incorporating the minmax approximation of peak strain energy density into the objective function led to a $44 \%$ reduction in its maximum value during the gait cycle. This reduction was realized near toe-off (just under $60 \%$ of the cycle) and occurred in the plantar tissue directly under the metatarsal heads (Fig. 3). As might be expected, the "strain" optimization caused the RMS values for kinematic tracking to increase slightly to $2.3^{\circ}, 2.1^{\circ}$, and $3.3^{\circ}$ for hip, knee, and ankle angles due to the incorporation of the additional term in the objective (Fig. 2). Muscle activation patterns displayed important differences between the two objectives. In order to reduce the peak strain near toe-off, the "strain" solution altered the magnitudes of the soleus, gastrocnemius, and tibialis anterior muscles (Fig. 4), possibly to restrict ankle plantar flexion to reduce the internal forefoot deformation at about $60 \%$ of the gait cycle (Fig. 3 ). This difference, while leading to a significant drop in peak strain energy, reduced the peak contact pressure by only $7 \%$ and ground reaction force by $26 \%$, at the same point in the cycle (Fig. 5). Of note, the temporal location of peak strain energy did not coincide with the maximum GRF and contact pressure locations, which occurred at either $\sim 15 \%$ or $\sim 50 \%$ of the cycle, depending on the solution (Fig. 5 ). This result is due to the relatively thin layer of plantar tissue in the toes and forefoot where internal tissue deformations can be high even for lower external loads.

Computation time ranged from 14 days for the "tracking" solution to 10 days for the "strain" solution using a single processor $2 \mathrm{GHz}$ Pentium Xeon desktop machine. For both solutions, more than $99.5 \%$ of the computation time was spent solving the FE model.

\section{Discussion}

This study demonstrated that model-based prediction of gait adaptations to accommodate localized tissue relief is computationally feasible. Within an optimal control framework, direct collocation allowed coupled simulations of movement and tissue mechanics. Novelty was not only achieved by including a continuum model of tissue deformation in simulation of gait but also by incorporating an internal tissue deformation metric into the movement optimization objective. By simulating an entire gait cycle and requiring periodicity at a specified velocity, we avoided the trivial solutions which reduce tissue loading simply by reducing muscle activations and ground reaction forces. Any reduction in ground reaction force must be compensated by an increase elsewhere in the gait cycle to maintain the specified velocity and periodic movement. These constraints in the tissue loading optimization could only be achieved because a dynamic musculoskeletal model with optimal control was coupled to the finite element model.

The results specifically showed that relatively small changes in neuromuscular control for the "strain" solution resulted in minor kinematic changes but substantial reduction in peak strain energy density experienced by the plantar tissue by unloading the metatarsal heads. Sensitivity of the solution to the chosen objective function weight values (Eq. (4)) is recognized as well as the currently unknown method the nervous system uses to prioritize tissue strain or tracking, if it uses these metrics at all. We emphasize that such predictions still require validation with human experiments, both at the tissue level and the neuromuscular level.

Strain energy density, a local internal tissue deformation metric, reflects the amount of strain in a localized area and, for that optimization, results were only weakly corroborated by external contact pressures. Even with this simple FE foot model, internal tissue conditions did not reflect external loading or plantar pressure distribution. This discrepancy, due to the 

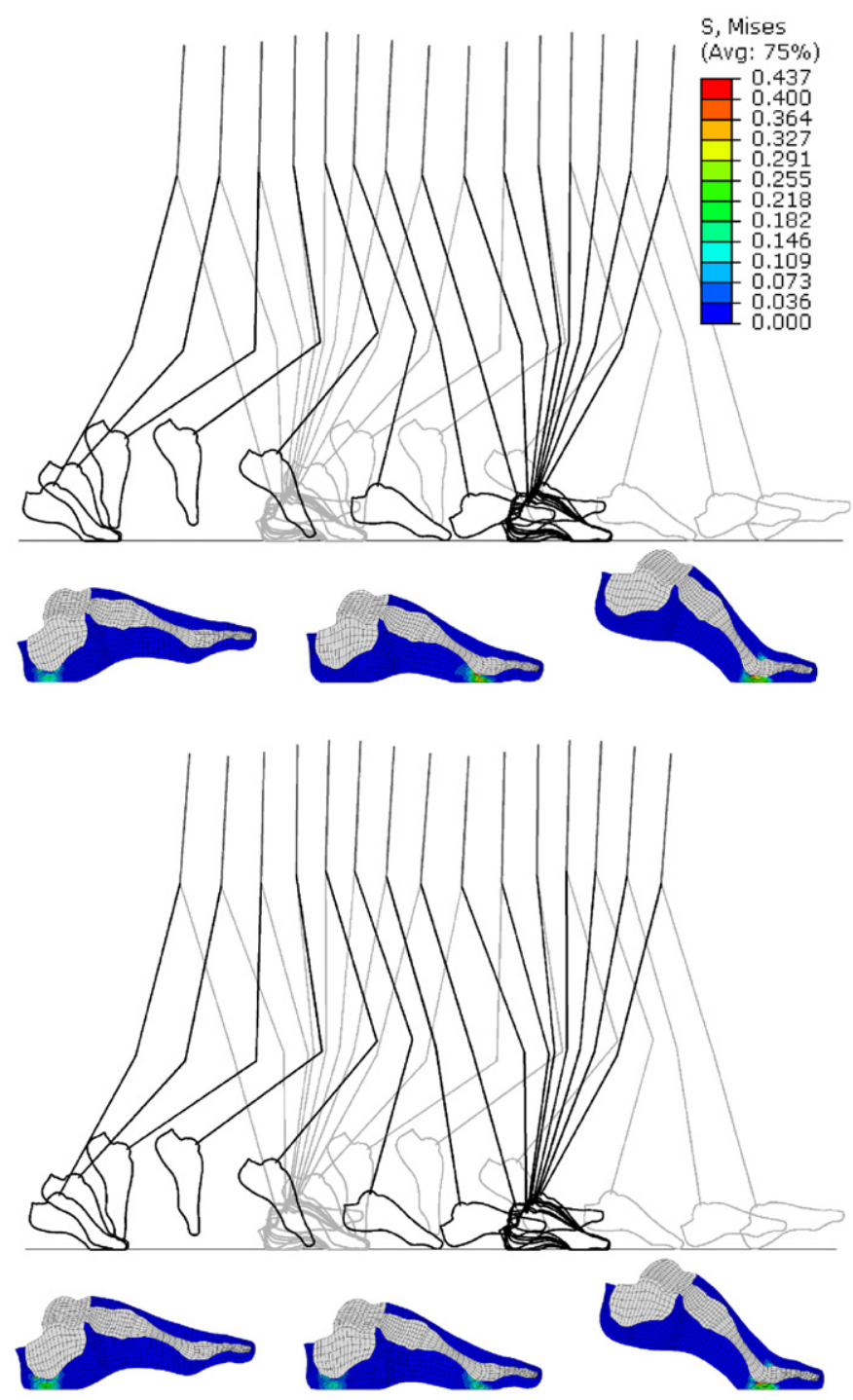

Fig. 1. Stick figure results for the "tracking" (top) and "strain" (bottom) solutions. An outline of the deformable finite element foot is shown with closeup von Mises stress distribution for heel-strike, mid-stance, and toe-off (left to right) below each stick figure. The mid-stance stress contours demonstrate how the "strain" solution modified the tissue loading during minimization of the peak strain energy density. The legend is consistent for all simulations and is in $\mathrm{MPa}$.

material response of soft tissue and the anatomical detail of foot structures, is highly relevant to the study of loading and contact conditions which could potentially lead to diabetic foot ulceration. Loads and pressures as typically measured in a gait lab may not necessarily reflect the extent of underlying tissue deformation. The preliminary findings and their clinical implications will be further explored after future model validation.

After this demonstration of methods and their feasibility, ongoing development and validation is necessary to produce accurate and valid 3D models from extensive data sets (Erdemir et al., 2009). A noteworthy limitation in the present work is the incorporation of friction. Due to the added complexity, friction was not defined explicitly in the FE contact but rather in the musculoskeletal system as a global measure. Also of note in the friction model, an approximation of the Coulomb behavior was chosen (Eq. (2)) to ensure continuity of the underlying model, a necessary characteristic to facilitate convergence when using gradient based optimization and direct collocation. Future work
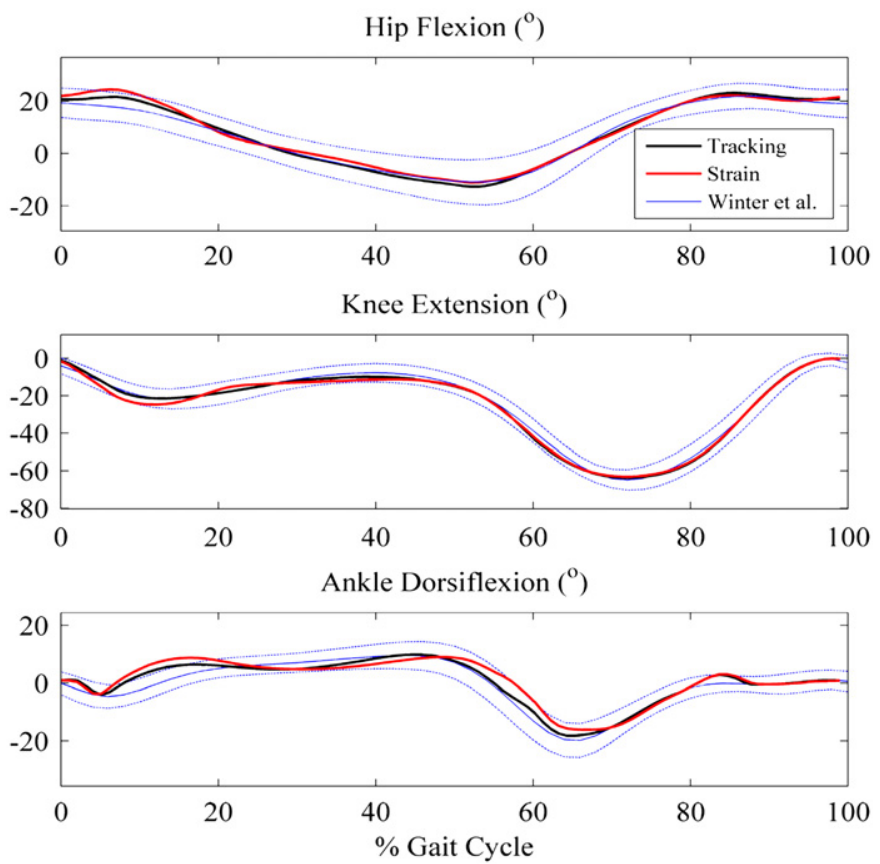

Fig. 2. Hip, knee, and ankle angles as a function of gait cycle. Experimental data (Winter, 1991) includes one standard deviation from the mean, represented by the dashed lines.

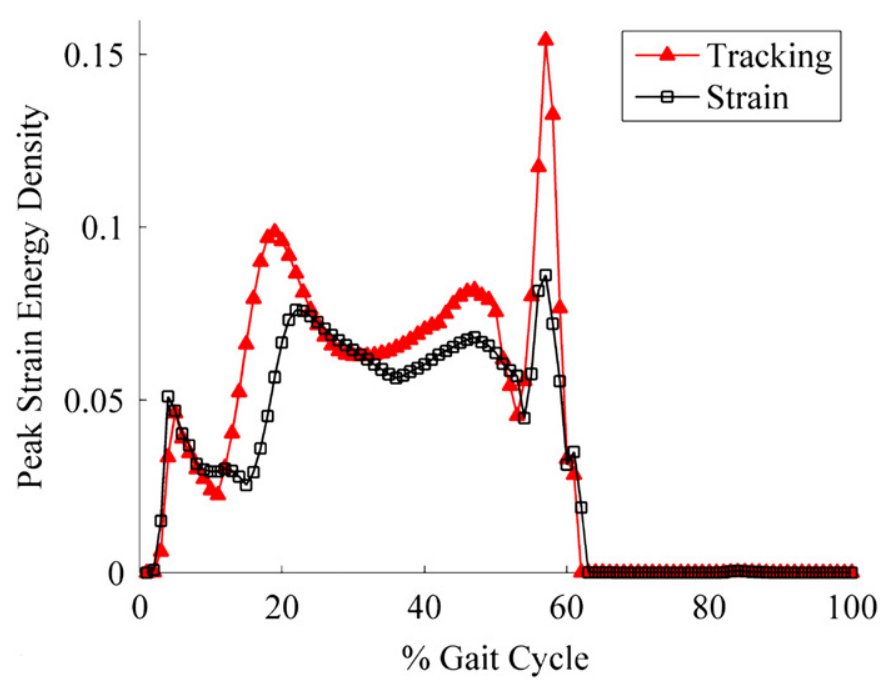

Fig. 3. Peak strain energy density as a function of percent gait cycle. $0 \%$ represents heel-strike while toe-off occurs at approximately $60 \%$ of the cycle. The physical location of peak strain energy density occurred at progressive locations in the plantar tissue throughout the cycle, from the heel to the toe. The maximum value during the cycle occurred just before toe-off and in the plantar tissue directly under the metatarsal heads.

will include implementing a more realistic and local friction model.

Computational expense may still be a concern, even for this relatively simple planar model. It should be noted that the same optimization with a simple spring-damper ground contact model, required less than one hour to solve (Ackermann and van den Bogert, 2010), pointing towards FE simulations as the computational bottleneck. This issue will be confounded with increased complexity and will be addressed in future work through the use 


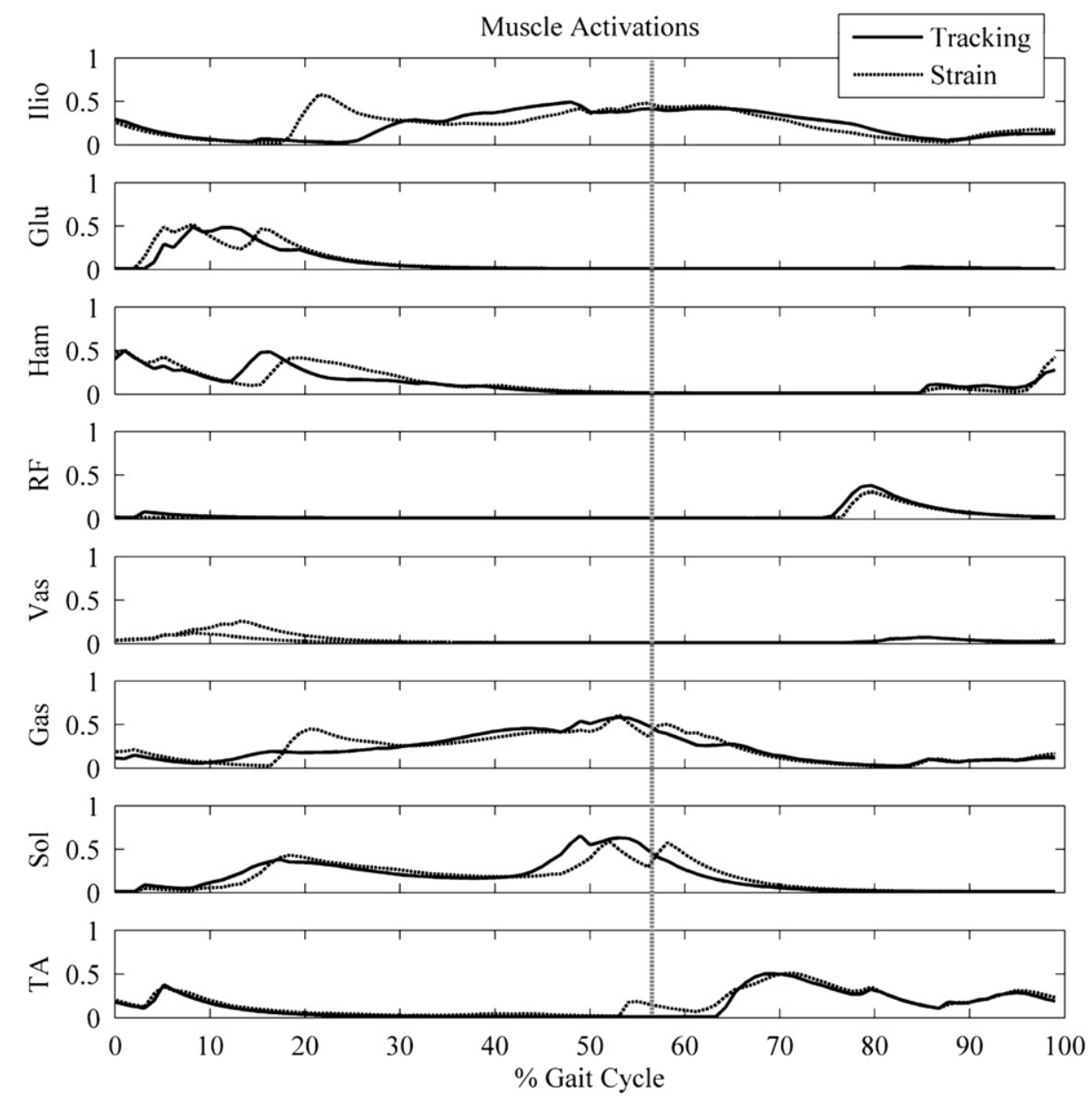

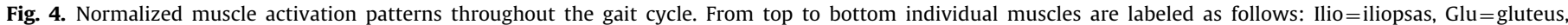

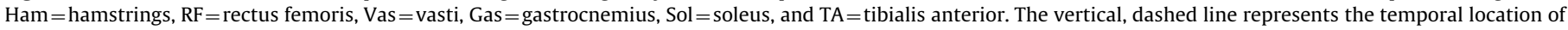
maximum strain energy density for the "tracking" solution (Fig. 3).

of a previously developed surrogate modeling method (Halloran et al., 2009). With this method, interpolation is performed on previous results to reduce the need for computationally expensive FE simulations. Computation time may still depend on many other factors but given the temporal setup of the direct collocation method, parallel processing with multiple CPU cores is possible.

Previous studies have demonstrated the importance of a reasonable initial guess when using direct collocation (Ackermann and van den Bogert, 2010). The initial guess for the "tracking" solution found in this study was adopted from the work by Ackermann and van den Bogert (2010). To minimize the number of iterations, the "strain" optimization used the "tracking" simulation as its initial guess. The computation times show that the "strain" optimization indeed required fewer iterations to arrive at convergence. Sensitivity of the results to the initial guess and formulation of the objective should be evaluated but results remain encouraging and robustness was demonstrated through the successful implementation of multiple objectives. Solutions were able to predict different motor control patterns while providing the underlying tissue, joint, and muscular loading. It is worth noting that the problem could have also been framed without including a tracking term in the objective. Alternative gait patterns could thus be explored in future studies.

After sufficient validation, multidomain simulations such as these have many potential clinical applications. The minimization of peak tissue strain is relevant for mechanically induced foot complications such as diabetic ulcers, but the methodology is not limited to the foot. A coupling of a musculoskeletal movement simulation with a knee joint model could lend insight into the mechanical and neuromuscular mechanisms that contribute to osteoarthritis. The complex interaction between muscular loading and soft tissue restraint present in the spinal column would also be a worthwhile framework to develop, to provide a scientific basis for the relationship between neuromuscular control and back pain. The approach may also be extended to include coupling tissue level loading with cell mechanics to predict biological responses to cell deformation. Obviously the possibilities are numerous and the methods are being developed as general modeling tools, to be applied as the need arises.

The presented modeling framework represents a worthwhile advancement in modeling capabilities. This is the first study to model gait with a concurrent coupled simulation of musculoskeletal and tissue deformation models. This study was also the first to successfully incorporate a local tissue deformation metric into the objective of a movement optimization. This advancement opens the door to explore the interactions between tissue deformation, muscle forces and control, kinematics, and external loading. This type of work is necessary to help in advancing the capability and clinical relevance of predictive movement simulations and it is the hope of the authors that the methods will be adopted and expanded on by the research community. 

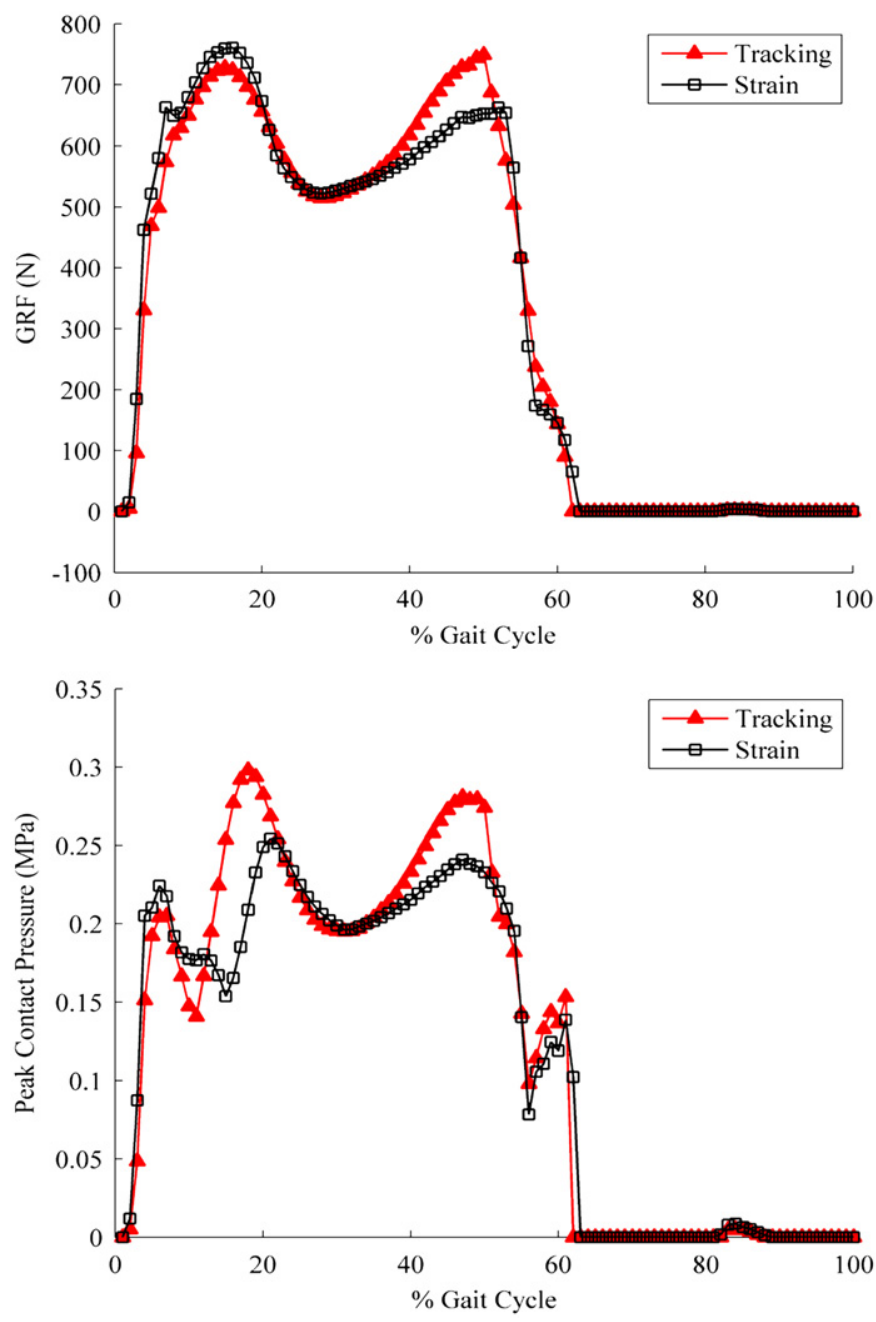

Fig. 5. Ground reaction force (GRF) and peak contact pressure as a function of percent gait cycle. $0 \%$ represents heel-strike while toe-off occurs at approximately $60 \%$ of the cycle.

\section{Conflict of interest statement}

The authors have no conflicts of interest to report.

\section{Acknowledgments}

This study is supported by the National Institutes of Health Grant R01 EB006735. The authors would like to thank Scott Sibole for developing the initial finite element mesh of the foot.

\section{References}

Ackermann, M., \& van den Bogert, A.J., 2010. Optimality principles for model-based prediction of human gait. Journal of Biomechanics 43, 1055-1060.

Anderson, F.C., Pandy, M.G., 2001. Dynamic optimization of human walking. Journal of Biomechanical Engineering 123, 381-390.

Besier, T.F. Gold, G.E., Beaupré, G.S., Delp, S.L., 2005. A modeling framework to estimate patellofemoral joint cartilage stress in vivo. Medicine and Science in Sports and Exercise 37, 1924-1930.
Boden, B.P., Dean, G.S., Feagin, J.A., Garrett, W.E., 2000. Mechanisms of anterior cruciate ligament injury. Orthopedics 23, 573-578.

Cattaneo, P.M., Kofod, T., Dalstra, M., Melsen, B., 2005. Using the finite element method to model the biomechanics of the asymmetric mandible before, during and after skeletal correction by distraction osteogenesis. Computer Methods in Biomechanics and Biomedical Engineering 8, 157-165.

Crowninshield, R.D., Brand, R.A., 1981. A physiologically based criterion for muscle force prediction in locomotion. Journal of Biomechanics 14, 793-801.

El-Rich, M., Shirazi-Adl, A., 2005. Effect of load position on muscle forces, internal loads and stability of the human spine in upright postures. Computer Methods in Biomechanics and Biomedical Engineering 8, 359-368.

Erdemir, A., McLean, S.G., Herzog, W., van den Bogert, A.J., 2007. Model-based estimation of muscle forces exerted during movements. Clinical Biomechanics (Bristol, Avon) 22, 131-154.

Erdemir, A., Sirimamilla, P.A., Halloran, J.P., van den Bogert, A.J., 2009. An elaborate data set characterizing the mechanical response of the foot. Journal of Biomechanical Engineering 131, 094502.

Erdemir, A., Viveiros, M.L., Ulbrecht, J.S., Cavanagh, P.R., 2006. An inverse finiteelement model of heel-pad indentation. Journal of Biomechanics 39, 1279-1286.

Fernandez, J.W., Pandy, M.G., 2006. Integrating modelling and experiments to assess dynamic musculoskeletal function in humans. Experimental Physiology 91, 371-382.

Gerritsen, K.G., van den Bogert, A.J., Hulliger, M., Zernicke, R.F., 1998. Intrinsic muscle properties facilitate locomotor control-a computer simulation study. Motor Control 2, 206-220.

Halloran, J.P., Erdemir, A., van den Bogert, A.J., 2009. Adaptive surrogate modeling for efficient coupling of musculoskeletal control and tissue deformation models. Journal of Biomechanical Engineering 131, 011014.

Hopkins, A.R., Hansen, U.N., Amis, A.A., 2005. Finite element models of total shoulder replacement: application of boundary conditions. Computer Methods in Biomechanics and Biomedical Engineering 8, 39-44.

Huiskes, R., Hollister, S.J., 1993. From structure to process, from organ to cell: recent developments of FE-analysis in orthopaedic biomechanics. Journal of Biomechanical Engineering 115, 520-527.

Jones, A.C., Wilcox, R.K., 2008. Finite element analysis of the spine: towards a framework of verification, validation and sensitivity analysis. Medical Engineering \& Physics 30, 1287-1304.

Koolstra, J.H., van Eijden, T.M.G.J., 2005. Combined finite-element and rigid-body analysis of human jaw joint dynamics. Journal of Biomechanics 38, 2431-2439.

Lin, Y., Haftka, R.T., Queipo, N.V., Fregly, B.J., 2009. Two-dimensional surrogate contact modeling for computationally efficient dynamic simulation of total knee replacements. Journal of Biomechanical Engineering 131, 041010.

Markolf, K.L., O’Neill, G., Jackson, S.R., McAllister, D.R., 2004. Effects of applied quadriceps and hamstrings muscle loads on forces in the anterior and posterior cruciate ligaments. The American Journal of Sports Medicine 32, 1144-1149.

McLean, S.G., Huang, X., Su, A., van den Bogert, A.J., 2004. Sagittal plane biomechanics cannot injure the ACL during sidestep cutting. Clinical Biomechanics (Bristol, Avon) 19, 828-838.

McLean, S.G., Su, A., van den Bogert, A.J., 2003. Development and validation of a 3-D model to predict knee joint loading during dynamic movement. Journal of Biomechanical Engineering 125, 864-874.

Neptune, R.R., 2000. Computer modeling and simulation of human movement. Applications in sport and rehabilitation. Physical Medicine and Rehabilitation Clinics of North America 11, 417-434

Rasmussen, J., Damsgaard, M., Voigt, M., 2001. Muscle recruitment by the min/max criterion-a comparative numerical study. Journal of Biomechanics 34, 409-415.

Shimokochi, Y., Yong Lee, S., Shultz, S.J., Schmitz, R.J., 2009. The relationships among sagittal-plane lower extremity moments: implications for landing strategy in anterior cruciate ligament injury prevention. Journal of Athletic Training 44, 33-38

van den Bogert, A.J., 1994. Analysis and simulation of mechanical loads on the human musculoskeletal system: a methodological overview. Exercise and Sport Sciences Reviews 22, 23-51.

Veves, A., Murray, H.J., Young, M.J., Boulton, A.J., 1992. The risk of foot ulceration in diabetic patients with high foot pressure: a prospective study. Diabetologia 35 , 660-663.

Winter, D., 1991. The Biomechanics and Motor Control of Human Gait: Normal, Elderly and Pathological. University of Waterloo Press, Waterloo.

Zajac, F.E., Neptune, R.R., Kautz, S.A., 2003. Biomechanics and muscle coordination of human walking: part II: lessons from dynamical simulations and clinical implications. Gait \& Posture 17, 1-17.

Zhang, M., Mak, A.F., Roberts, V.C., 1998. Finite element modelling of a residual lower-limb in a prosthetic socket: a survey of the development in the first decade. Medical Engineering \& Physics 20, 360-373. 